\title{
Hidden Markov Model
}

National Cancer Institute

\section{Source}

National Cancer Institute. Hidden Markov Model. NCI Thesaurus. Code C75940.

A statistical model in which the system being modeled has unknown parameters, but the hidden parameters can be determined from the observable parameters. While the state is not directly visible, the variables influenced by the state are visible. Each state has a probability distribution over the possible output tokens. Therefore the sequence of tokens generated by a Hidden Markov Model (HMM) gives some information about the sequence of states. 